Mitochondrial neurogastrointestinal encephalomyopathy in an 18-year old female presented with symptoms suggestive of relapsing CIDP, but the gastrointestinal symptoms and lactic acidosis were not compatible (Ginsberg L et al. The Lancet Neurology February 2007;6:192-198).

Noninvasive ventilation used in treatment of respiratory failure in children with neuromuscular disease results in a reduction of symptoms, hospitalizations, and health care costs, without adverse effects on quality of life. Fourteen patients were followed for 6 to 84 months (median $30 \mathrm{mo}$ ). Daytime sleepiness and headache, and sleep quality improved, despite disease progression. (Young HK et al. Neurology 2007;68:198-201).

\title{
MOVEMENT DISORDERS
}

\section{MOVEMENT DISORDERS INDUCED BY ANTIEPILEPTIC DRUGS}

The prevalence and risk factors for the development of movement disorders, mainly parkinsonism and postural tremor, were analyzed in 201 adults ( $>18$ years, mean 40 years) treated with valproate and newer antiepileptic drugs (AED) at the Epilepsy Clinic, Toronto Western Hospital, Ontario, Canada. Postural tremor was the most common movement disorder, occurring in $45 \%$ of patients. Tremor was mild in $70 \%$ and moderate to severe in $30 \%$. Valproate and carbamazepine were most commonly associated with tremor, followed by phenytoin. Parkinsonism developed in $4.5 \%$, and the odds of having parkinsonism was 5 times higher with valproate than with other AEDs. Five of 9 patients with parkinsonism were concurrently using other drugs or had comorbidities, that could have caused or exacerbated the parkinsonism. Dystonia diagnosed in 4 patients presented with writer's cramp (with carbamazepine, clobazam and topiramate in 1, carbamazepine and valproate in 1), and foot inversion and hand posturing in 1 with a history of cerebral palsy (with topiramate). Tics manifested by excessive blinking developed in 2 patients, one on a combination of phenytoin and clobazam, and the other on valproate. None of the newer AEDs was clearly associated with movement disorders, and these included lamotrigine, vigabatrin, levetiracetam, and oxcarbazepine, but the numbers were small. (Zadikoff C, Munhoz RP, Asante AN et al. Movement disorders in patients taking anticonvulsants. J Neurol Neurosurg Psychiatry February 2007;78:147-151). (Respond: Dr C Zadikoff, Division of Neurology, Toronto Western Hospital, University of Toronto, Toronto, Ont, Canada).

COMMENT. Postural tremor is a common side effect of AEDs in adults and especially with valproate (VPA). In children, VPA also causes tremor and choreiform movements (Lancman ME et al. Arch Neurol 1994;51:702-704). The chorea was dose related; it resolved when VPA was withdrawn or replaced by divalproex sodium sprinkles. Reversible dementia and brain atrophy are reported during VPA therapy in 2 children ages 8 and 10 years. (Papazian O et al. Ann Neurol 1995;38:687-691). 